\title{
Observing Misfit Dislocation Interactions Across Thin Film Oxide Heterostructures
}

\author{
Everett D. Grimley ${ }^{1}$, Edward Sachet ${ }^{1}$, Brian F. Donovan ${ }^{2}$, Patrick E. Hopkins ${ }^{2}$, Jon-Paul Maria ${ }^{1}$ and \\ James M. LeBeau ${ }^{1}$ \\ 1. Department of Materials Science and Engineering, North Carolina State University, Raleigh, USA \\ 2. Department of Mechanical and Aerospace Engineering, University of Virginia, Charlottesville, USA
}

Complete realization of next-generation plasmonic devices has been plagued by the high dielectric losses and the limited spectral regime of traditional noble metal plasmonic materials. Oxide semiconductors are poised to be suitable alternatives to traditional plasmonic materials, as oxides possess both low dielectric losses and tunable band gaps which are important properties for enhancing plasmonic performance. Recently, Sachet et al. showed that dysprosium doping of thin film $\mathrm{CdO}$ affords dramatic control over the film's optical properties by altering the carrier concentration and carrier mobility [1]. This exciting discovery indicates that $\mathrm{Dy}: \mathrm{CdO}$ is a suitable material for tunable use in the mid-IR plasmonic regime. $\mathrm{CdO}$ films are epitaxially grown on $\mathrm{MgO}$ substrates and possess a nearly $12 \%$ lattice mismatch with the substrate. Such a misfit is expected to be accompanied by interfacial dislocations to reduce strain. These dislocations and their associated strain fields are expected to play a further role in altering film conductivity and thermal properties, as they form scattering centers for charge carriers and phonons. This effect is expected to be more pronounced for thinner films.

To examine the interplay between $\mathrm{CdO}$ film thermal properties and interfacial dislocations/strain, a thickness series of sub-10 nm CdO films were deposited on (001)-oriented $\mathrm{MgO}$ substrates and were capped with $10 \mathrm{~nm}$ of $\mathrm{MgO}$. Dislocation ordering and the associated strain fields at top and bottom $\mathrm{CdO} / \mathrm{MgO}$ interfaces were analyzed with scanning transmission electron microscopy (STEM). Micrographs were acquired using the RevSTEM technique to minimize drift distortion [2]. The images were acquired using low-angle annular dark-field (LAADF)-STEM with a probe-corrected FEI Titan G2 60-300 kV microscope operated at $200 \mathrm{kV}$ with detector inner semi-angle and probe semi-convergence angles of approximately $34 \mathrm{mrad}$ and 19.6 respectively, and with a beam current of approximately 40 pA. The RevSTEM series were acquired as sets of 40 frames sized 1024x1024 pixel with a $2 \mu$ s/pixel dwell time and a $90^{\circ}$ scan rotation between successive frames.

For all film thicknesses in the series, edge dislocations with $<110>$ oriented lines and $1 / 2<110>$ type Burgers vectors were observed at both top and bottom $\mathrm{CdO} / \mathrm{MgO}$ interfaces, and the dislocations were formed by the $<111>$ type planes as is common rock salt oxides (see Figure 1 a) $-\mathrm{c}$ )). Based on the lattice misfit in the direction of the Burgers vector, dislocations were expected to occur every 2.7 to 3.7 $\mathrm{nm}$. Dislocation spacing for films in the thickness series were observed to fall within the calculated range of spacing, though dislocation spacing and arrangement showed dependence on film thickness, especially for the thinner films. Geometric phase analysis (GPA) [3] showed the formation of complex strain fields in the thinner $\mathrm{CdO}$ films due to strain field interactions between dislocations on opposite $\mathrm{CdO} / \mathrm{MgO}$ interfaces (as in Figure $2 \mathrm{~b}$ )). These transverse dislocation interactions alter the strain fields in the interior of the $\mathrm{CdO}$ films which could dramatically impact the properties of the thinner films. By contrast, this overlap and strain field array formation is not present for thicker films (as in Figure 2 d)) where spacing and arrangement of dislocations depends primarily on misfit. These findings directly show transverse interaction between dislocations in heterostructures. Finally, we will discuss the role of these dislocations on controlling film properties, in particular thermal and electrical conductivity [4]. 


\section{References:}

[1] E Sachet et al, Nature Materials 14 (2015), p. 414.

[2] X Sang and JM LeBeau, Ultramicroscopy 138 (2014), p. 28.

[3] FRWRtools plugin for Digital Micrograph by Christoph T. Koch (Humboldt-Universität zu Berlin)

[4] The authors greatly acknowledge funding from the National Science Foundation through a Career Award (DMR 1350273) and through the National Science Foundation Graduate Research Fellowship Program (DGE 1252376). The authors acknowledge use of Analytical Instrumentation Facility at North Carolina State University, which is supported by the State of North Carolina and by the National Science Foundation.

1. a)

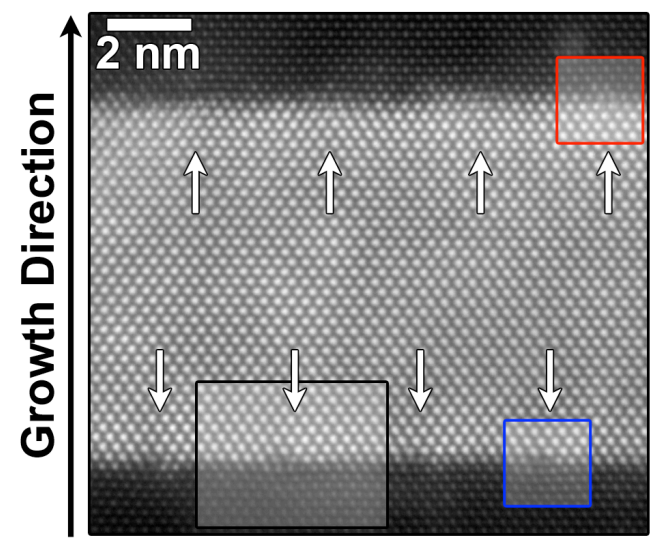

b)

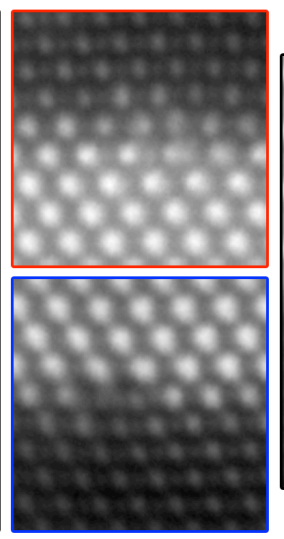

c)

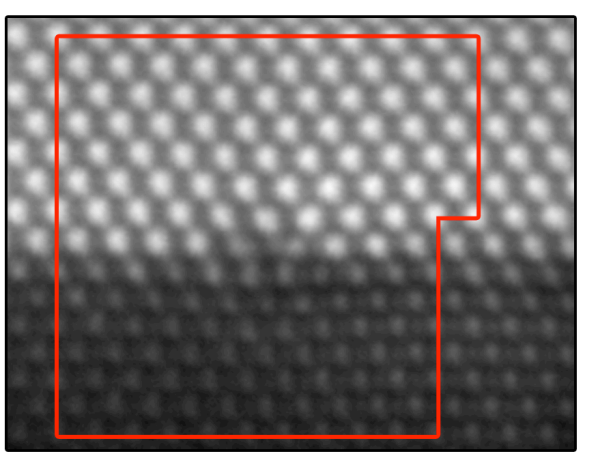

Figure 1. a) LAADF-STEM image of $9 \mathrm{~nm}$ thick $\mathrm{CdO}$ film viewed down the [110] zone-axis with arrows pointing to misfit dislocations at the $\mathrm{CdO} / \mathrm{MgO}$ interfaces and with insets showing the regions expanded in b) and c). b) Dislocations at both interfaces have lines in the [110] directions and are formed by the $<111>$ type planes. c) Burgers circuit drawn showing that the Burgers vectors lie in $1 / 2$ a $<110>$ directions.

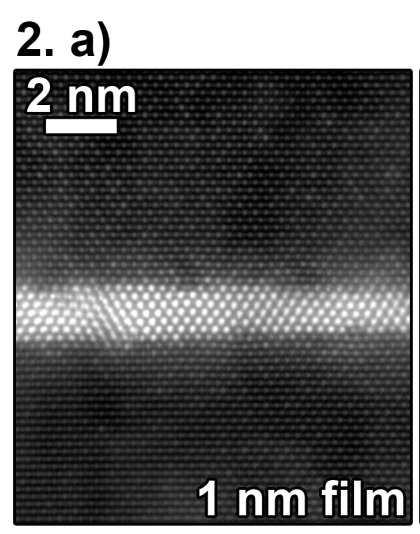

b)

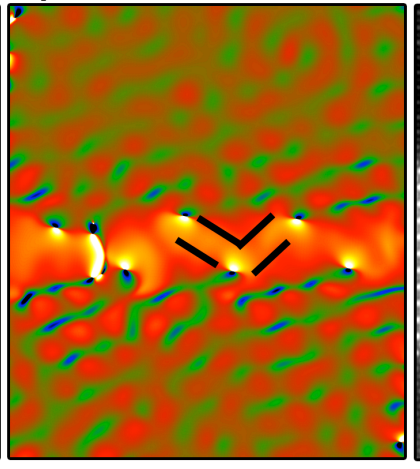

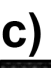

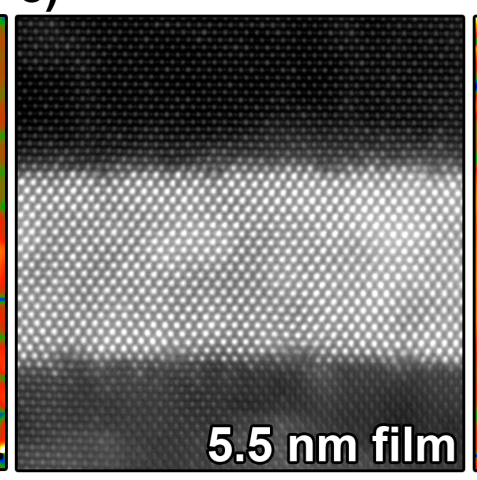

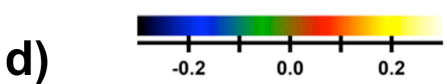

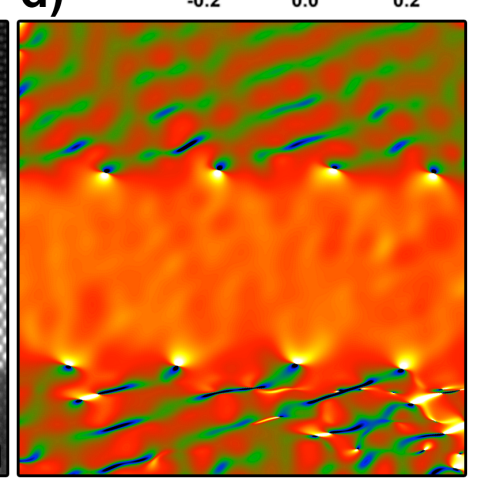

Figure 2. LAADF-STEM images of a) $1 \mathrm{~nm} \mathrm{c}$ ) and $5.5 \mathrm{~nm}$ films. b) GPA of of the $1 \mathrm{~nm}$ film (strain scale shown above d)) shows formation of a zigzagging strain field (partially outlined with black lines) which arises due to overlap in the film interior of strain fields that originate from dislocations on opposite interfaces. In contrast, transverse dislocation interaction is absent from thicker films, such as the $5.5 \mathrm{~nm}$ film in d). Complex strain field formation such as in b) is expected to impact properties in the thinner films. All images are at equal magnification. Image levels are adjusted to enhance contrast. 